\title{
QUADRATIC ADDITION THEOREMS FOR EVEN FUNCTIONS*
}

\author{
BY J. D. GRANT
}

The purpose of this paper is to show that if an even function be expansible in power series in some region about the origin, and satisfy a quadratic addition $\dagger$ theorem, the function is a linear fractional transformation of the Weierstrass " $P$ " function or one of its degenerate forms.

P. D. Edwards $\ddagger$ has shown that addition theorems are symmetric in $U=F(x)$ and $V=F(y)$. He has also shown the most general form of an addition theorem linear in $W=F(x+y)$. In this paper, equations quadratic in $W$ are treated by means of the following theorem.

THEOREM 1. An addition theorem for an even function is symmetric in the three variables $U, V, W$.

For one may set

$$
\begin{aligned}
& x+y=s, \quad W=F(x+y)=F(s) \quad=U^{\prime}, \\
& x=s+t, U=F(x) \quad=F(s+t) \quad=W^{\prime}, \\
& y=-t, \quad V=F(y) \quad=F(-t)=F(t)=V^{\prime} .
\end{aligned}
$$

Thus the equation, which is known to be symmetric in $U, V$, is shown to be symmetric in $U, W$ so that the theorem follows.

In the same manner one easily proves the following companion theorem for odd functions.

Theorem 2. An addition theorem for an odd function is symmetric in the three variables $U, V,-W$.

* Presented to the Society, April 3, 1931.

$\dagger$ An algebraic addition theorem is a polynomial relation between $F(x)$, $F(y)$, and $F(x+y)$, with constant coefficients. For this paper the polynomial is irreducible. Weierstrass made addition theorems the basis for his study of elliptic functions. Osgood, Allgemeine Funktionentheorie, 1912, vol. 1, pp. 580595; Forsyth, Theory of Functions, 1918, Chapter XIII; Hancock, Theory of Elliptic Functions, 1910.

$\ddagger$ Abstract No. 8, this Bulletin, vol. 35 (1929), p. 453. The paper is available at the University of Indiana Library. 
This theorem is illustrated by $\tan x$ and $\operatorname{ctn} x$, but the form is lost for $\sin x$ and Jacobi's sn $x$, whose addition theorems are symmetric in $U^{2}, V^{2}, W^{2}$.

The equation whose solution we wish to study is the following, symmetric in the three variables $U, V, W$ :

$$
\begin{aligned}
& a_{0}+a_{1}(U+V+W)+a_{2}(U V+U W+V W) \\
& \quad+a_{3}\left(U^{2}+V^{2}+W^{2}\right)+a_{4} U V W \\
& \quad+a_{5}\left(U V^{2}+U^{2} V+U W^{2}+U^{2} W+V W^{2}+V^{2} W\right) \\
& \quad+a_{6}\left(U^{2} V^{2}+U^{2} W^{2}+V^{2} W^{2}\right)+a_{7} U V W(U+V+W) \\
& \quad+a_{8} U V W(U V+U W+V W)+a_{9} U^{2} V^{2} W^{2}=0 .
\end{aligned}
$$

This may also be written in powers of $W$ as

$$
R_{1}(U, V) W^{2}-2 R_{2}(U, V) W+R_{3}(U, V)=0,
$$

where $R_{1}, R_{2}, R_{3}$ are of degree not more than two in $U$ or $V$.

Since we are dealing with even functions, changing $y$ to $-y$ alters only $W$. Hence the two roots of this quadratic are $W=F(x+y), F(x-y)$, and by the ordinary theorems regarding symmetric functions of the roots one has

(3) $F(x+y)+F(x-y)=2 R_{2} / R_{1}, F(x+y) \cdot F(x-y)=R_{3} / R_{1}$.

Thus two other interesting types of functional equations are seen to arise naturally and will be solved when $R_{1}, R_{2}, R_{3}$ are quadratics symmetric in $U, V$.

It has been shown* that, if the first term of the power series expansion of $F(x)$ is not a constant, there is in the functional equation a group of terms which form an equation having the first term of the series as a solution. Since first terms of the form $c_{n} x^{n}, n<-2$, or $n>2$, would require the vanishing of all the coefficients $a$ in (1), we have to consider only three possible first terms, $c_{-2} x^{-2}, c_{0}, c_{2} x^{2}$. In case the first term is $c_{0}$, one writes

$$
U=U_{1}+c_{0}
$$

and the first term for $U_{1}$ will be $c_{2} x^{2}$. In case the first term is $c_{2} x^{2}$, one writes

$$
U=1 / U^{\prime}
$$

* The author in American Mathematical Monthly, vol. 37, p. 70. 
and the first term for $U^{\prime}$ will be $c_{-2} x^{-2}$. The transformed equation in $U_{1}, V_{1}, W_{1}$, or $U^{\prime}, V^{\prime}, W^{\prime}$ will still be of type (1). Hence we see that any solution of an equation (1) is a linear fractional transformation of a solution of a type (1) equation, whose series expansion begins with $c_{-2} x^{-2}, c_{-2} \neq 0$. For this reduced case, equation (1) contains the terms of the addition theorem for $x^{-2}$ as its terms of highest degree and they must enter with a nonzero multiplier (chosen as unity). This gives our equation (1) as

$$
\begin{aligned}
& a_{0}+a_{1}(U+V+W)+a_{2}(U V+U W+V W)+a_{3}\left(U^{2}+V^{2}+W^{2}\right) \\
& +a_{4} U V W+a_{5}\left(U V^{2}+U^{2} V+U W^{2}+U^{2} W+V W^{2}+V^{2} W\right) \\
& +U^{2} V^{2}+U^{2} W^{2}+V^{2} W^{2}-2 U V W(U+V+W)=0 .
\end{aligned}
$$

The solution has the expansion

$$
U=F(x)=\frac{1}{x^{2}}+c_{0}+c_{2} x^{2}+c_{4} x^{4}+\cdots
$$

For $x=y$, the roots of (2) become

$$
F(2 x), \quad F(0)=\infty, \quad \text { and } \quad R_{1}(U, U) \equiv 0,
$$

so that $a_{5}=a_{3}=0$. Equation (1) is further reduced to

(4) $a_{0}+a_{1}(U+V+W)+a_{2}(U V+U W+V W)+a_{4} U V W$

$$
+U^{2} V^{2}+U^{2} W^{2}+V^{2} W^{2}-2 U V W(U+V+W)=0 \text {. }
$$

Equation (4) is invariant in form under the transformation $U=U^{\prime}+t$ and the cubic term becomes $\left(a_{4}-12 t\right) U^{\prime} V^{\prime} W^{\prime}$. When $t=a_{4} / 12$, equation (4) becomes, if we omit accents,

(5) $b_{0}+b_{1}(U+V+W)+b_{2}(U V+V W+U W)$

$$
+U^{2} V^{2}+U^{2} W^{2}+V^{2} W^{2}-2 U V W(U+V+W)=0 \text {. }
$$

If we set $x=y$ in (5), substitute the power series for the solution, and after collecting terms equate to zero the coefficients of each power of $x$, the first four conditions on $c_{i}$ are:

$$
\begin{aligned}
& x^{-6}: \quad c_{0}=0, \\
& x^{-4}: 10 c_{2}-b_{2}=0, \\
& x^{-2}: 28 c_{4}-b_{1}=0, \\
& x^{0}: \quad 2 b_{0}+21 b_{2} c_{2}-90 c_{2}^{2}-510 c_{6}=0 .
\end{aligned}
$$


Again if we set $y=2 x$ in (5) and make the same computation, there results:

$$
\begin{aligned}
& x^{-6}: \quad c_{0}=0, \\
& x^{-4}: 10 c_{2}-b_{2}=0, \\
& x^{-2}: 28 c_{4}-b_{1}=0, \\
& x^{0}: 18 b_{0}+289 b_{2} c_{2}-1210 c_{2}^{2}-6390 c_{6}=0 .
\end{aligned}
$$

The conditions (6) and (7) require for consistency that $4 b_{0}=b_{2}^{2}$. To complete the proof one notices that if $b_{2}=g_{2} / 2$, $b_{1}=g_{3}, b_{0}=b_{2}^{2} / 4=g_{2}^{2} / 16$, the equation (1) has been reduced by means of linear fractional transformations to the well known addition theorem* for $P\left(x ; g_{2}, g_{3}\right)$. In this connection the degenerate forms of $P\left(x ; g_{2}, g_{3}\right)$ should be taken as $\left(2 / 3+\operatorname{ctn}^{2} x\right)$ and $x^{-2}$, as these have $c_{0}=0$.

It will be seen from a study of the addition theorem for $P\left(x ; g_{2}, g_{3}\right)$ that every second-degree addition theorem having an even solution may be written

$$
W=\frac{R_{2}(U V) \pm k U^{\prime} V^{\prime}}{R_{1}(U V)}
$$

where $U^{\prime}$ and $V^{\prime}$ are the derivatives of $U$ and $V$ with respect to $x$ and $y$ respectively.

\section{James Millikin University}

* Since the addition theorems give rise to the differential equation by a direct computation, (see Forsyth, p. 357), it is one of the many ways of defining the $P$ function. 